\section{Revisión sistemática cualitativa del uso de cuestionarios para el diagnóstico de ERGE en pediatría}

\author{
Hernández-Pliego RR ${ }^{1}$, Asbun-Bojalil J ${ }^{2}$, Anguiano-Robledo $\mathrm{L}^{3}$
}

Resumen

INTRODUCCIÓN: el uso de cuestionarios para identificar síntomas de enfermedad por reflujo gastroesofágico (ERGE) y su aplicación en población pediátrica es limitado, sobre todo en niños menores y lactantes, en los que resulta imposible conocer los síntomas debido a la incapacidad de manifestarlos de manera objetiva.

OBJETIVO: realizar una revisión sistemática de la literatura en población pediátrica con sospecha de ERGE, para evaluar el uso de cuestionarios como herramienta diagnóstica para esta enfermedad.

MATERIAL Y MÉTODOS: se realizó una búsqueda exhaustiva de la literatura en diversas bases de datos. La selección de los estudios fue realizada por dos investigadores expertos en el tema. La estrategia de búsqueda estuvo compuesta de las palabras clave: Gastroesophageal Reflux disease, Infant Gastroesophageal Reflux Questionnaire (IGERQ), infant, child, children, questionnaire. La calidad de los estudios fue evaluada con la escala Newcastle-Ottawa.

RESULTADOS: con la estrategia de búsqueda realizada fueron seleccionados tres estudios, los cuales utilizaron diferentes cuestionarios en poblaciones distintas: en 2006, Kleinman evaluó la confiabilidad del cuestionario IGER-Q en 185 pacientes menores de 18 meses obteniéndose 0.86-0.87; en 2005, Deal evaluó 64 pacientes de 1-11 meses y 63 pacientes de 1-4 años reportando una sensibilidad de 90 y $85 \%$ para cada grupo de edad, respectivamente y especificidad de 83 y $81.5 \%$, respectivamente; y Stordal, en 2005, evaluó 99 pacientes de 7-16 años con una sensibilidad de $75 \%$ y especificidad de $96 \%$.

CONCLUSIÓN: el uso de los cuestionarios como herramienta diagnóstica de ERGE en pacientes pediátricos es escaso y la evidencia clínica disponible no es suficiente para realizar una recomendación apropiada.

PALABRAS CLAVE: enfermedad de reflujo gastroesofágico (ERGE), niños, cuestionario, revisión sistemática.

\begin{abstract}
${ }^{1}$ Maestría en Ciencias. Sección de Estudios de Posgrado e Investigación. Laboratorio de Farmacología Molecular.

${ }^{2}$ Doctor en Investigación en Medicina. Sección de Estudios de Posgrado e Investigación.

${ }^{3}$ Doctorado en Investigación en Medicina. Sección de Estudios de Posgrado e Investigación. Laboratorio de Farmacología Molecular.

Escuela Superior de Medicina, Instituto Politécnico Nacional.
\end{abstract}

Recibido: 17 de agosto del 2015

Aceptado: 14 de enero del 2016

Correspondencia

Rogelio Ramsés Hernández-Pliego

Sección de Estudios de Posgrado e Investigación Laboratorio de Farmacología Molecular Escuela Superior de Medicina, Instituto Politécnico Plan de San Luis y Díaz Mirón s/n C.P. 11340 , Ciudad de México ram_geo@hotmail.com

Este artículo debe citarse como Hernández-Pliego RR, Asbun-Bojalil J, AnguianoRobledo L, . Revisión sistemática cualitativa del uso de cuestionarios para el diagnóstico de ERGE en pediatría. Acta Pediatr Mex. 2016;37(2):102-110. 


\section{Qualitative systematic review of the use of questionnaires for the diagnosis of GERD in pediatric}

\author{
Hernández-Pliego RR ${ }^{1}$, Asbun-Bojalil J², Anguiano-Robledo $\mathrm{L}^{3}$
}

Abstract

BACKGROUND/AIMS: The use of questionnaires to identify GERD symptoms in pediatric population is limited especially in young children and infants where it is impossible to objectively assess the symptomatology due saying helplessness. The aim of this study was to conduct a systematic review of the literature in pediatric patients with suspected GERD, to evaluate questionnaires usage as diagnostic tool for this disease.

MATERIAL AND METHODS: A comprehensive literature search in various databases was performed. Two expert researchers on the topic conducted the studies selection. The search strategy consisted of key words: Gastroesophageal Reflux disease, IGERQ, infant, child, children, and questionnaire. The studies quality was assessed using the Newcastle-Ottawa scale.

RESULTS: Following the searching strategy, there were selected three studies, which used different questionnaires in different populations, Kleinman 2006 evaluated 185 patients younger than 18 months with the IGER-Q, test reliability was evaluated yielding 0.86 to 0.87 , Deal 2005, 64 patients evaluated aged 1-11 months and 1-4 years and 63 patients reporting 90 and $85 \%$ sensitivity per age group respectively and 83 and $81.5 \%$ specificity respectively, and Stordal 2005 evaluated 99 patients aged 7-16 with 75\% sensitivity and 96\%specificity.

CONCLUSION: the use of questionnaires as diagnostic tool for GERD in pediatric patients is limited and available clinical evidence is not enough to make a proper recommendation.

KEYWORDS: Gastroesophageal reflux disease; children; questionnaire; systematic review
${ }^{1}$ Maestría en Ciencias. Sección de Estudios de Posgrado e Investigación. Laboratorio de Farmacología Molecular.

${ }^{2}$ Doctor en Investigación en Medicina. Sección de Estudios de Posgrado e Investigación.

${ }^{3}$ Doctorado en Investigación en Medicina. Sección de Estudios de Posgrado e Investigación. Laboratorio de Farmacología Molecular.

Escuela Superior de Medicina, Instituto Politécnico Nacional.

\section{Correspondence}

Rogelio Ramsés Hernández-Pliego

Sección de Estudios de Posgrado e Investigación

Laboratorio de Farmacología Molecular

Escuela Superior de Medicina, Instituto Politécnico

Plan de San Luis y Díaz Mirón s/n

C.P. 11340, Ciudad de México

ram_geo@hotmail.com

\section{INTRODUCCIÓN}

La enfermedad por reflujo gastroesofágico (ERGE) se presenta cuando el reflujo del contenido gástrico, sean sólidos o líquidos, causa síntomas molestos y/o complicaciones que afectan la calidad de vida de quienes la padecen. Es un trastorno gastrointestinal muy frecuente; se estima que $40 \%$ de los adultos tiene pirosis y/o regurgitación al menos una vez al mes; $20 \%$ una vez a la semana y $7 \%$ diariamente..$^{1-4}$ En niños es una enfermedad frecuente y la prevalencia de los síntomas varía con la edad..$^{5-7}$ Actualmente, el ERGE representa hasta un $65 \%$ de la consulta 
diaria del médico general o pediatra en el primer nivel de atención. ${ }^{6,8}$

Existen diversas formas de presentación clínica de la ERGE, los dos síntomas principales son la regurgitación y la pirosis; ${ }^{7,9}$ pero también puede presentarse vómito, disfagia, hematemesis, dispepsia. ${ }^{10}$ En niños es más frecuente la presencia de regurgitación que de pirosis, a diferencia del adultos que los dos síntomas son comunes. ${ }^{11}$ En ocasiones, la regurgitación suele acompañarse de vómito en proyectil. ${ }^{9,12}$

Debido a la gran diversidad de síntomas y signos que se pueden presentar en la ERGE, no existe una prueba diagnóstica considerada estándar de oro. ${ }^{13}$ El diagnóstico se realiza mediante una historia clínica y exploración física detalladas. ${ }^{9}$ Las guías clínicas de ERGE mencionan algunos estudios que pueden servir de apoyo para el diagnóstico: serie esófago-gastroduodenal (SEGD), pHmetría esofágica de 24 horas, ${ }^{14}$ endoscopia y gammagrafía. ${ }^{9,12}$ Las pruebas y estudios de laboratorio y gabinete se utilizan de acuerdo a la evaluación de cada paciente y dependiendo de los síntomas presentes, edad del paciente, recurrencia o la presencia de síntomas de alarma o complicaciones.

El uso de cuestionarios para la identificación de síntomas de ERGE en adultos ha sido bien documentado; ${ }^{15}$ sin embargo, su aplicabilidad en población pediátrica es limitada, sobre todo en niños menores y lactantes en los que resulta imposible conocer los síntomas por la incapacidad de manifestarlos de manera objetiva. ${ }^{16,17}$ Los cuestionarios tienen algunas ventajas: alta sensibilidad y especificidad, bajo costo, pueden ser aplicados en consultorio y no requieren de personal especializado. ${ }^{16}$

\section{OBJETIVO}

Realizar una revisión sistemática de la literatura en población pediátrica con sospecha de ERGE, para evaluar el uso de cuestionarios como herramienta diagnóstica para esta enfermedad y revisar si la edad de los pacientes influye en la sensibilidad y especificidad.

\section{MATERIAL Y MÉTODOS}

Se realizó una revisión sistemática utilizando el formato PICO (P: paciente o problema, I: intervención, C: intervención de comparación o control, O: out come o resultado) para la pregunta de investigación, la cual es la siguiente ¿el uso de cuestionarios en pacientes pediátricos con síntomas de ERGE es una herramienta sensible y específica para establecer el diagnóstico de la misma?

\section{Estrategia de búsqueda}

Se realizó una búsqueda exhaustiva de la literatura en el periodo comprendido del 1 de enero de 1980 al 4 de junio de 2015 en las siguientes bases de datos: Medline/Pubmed, Biblioteca Cochrane (CENTRAL), EMBASE, Imbiomed, LILACS, Artemisa y Nieto Editores. También se hizo una búsqueda manual en el buscador Google.

La estrategia de búsqueda estuvo compuesta de vocabulario controlado (Medical Subject Headings $[\mathrm{MeSH}]$ ) y lenguaje libre, utilizando las siguientes palabras clave: Gastroesophageal Reflux disease, Infant Gastroesophageal Reflux Questionnaire (IGERQ), infant, child, children, questionnaire (véase el Cuadro 1). La sintaxis se complementó utilizando operadores booleanos y cercanos. Esta estrategia se adaptó a todas las bases de datos. Se utilizaron los filtros de idioma (inglés y español), ensayos clínicos, meta-análisis y revisiones sistemáticas, siempre y cuando fuera posible utilizarlos en las bases de datos.

\section{Selección de los estudios}

La selección de los estudios fue realizada por dos investigadores expertos en el tema, seleccio- 
Cuadro 1. Ejemplo de la descripción del algoritmo de búsqueda

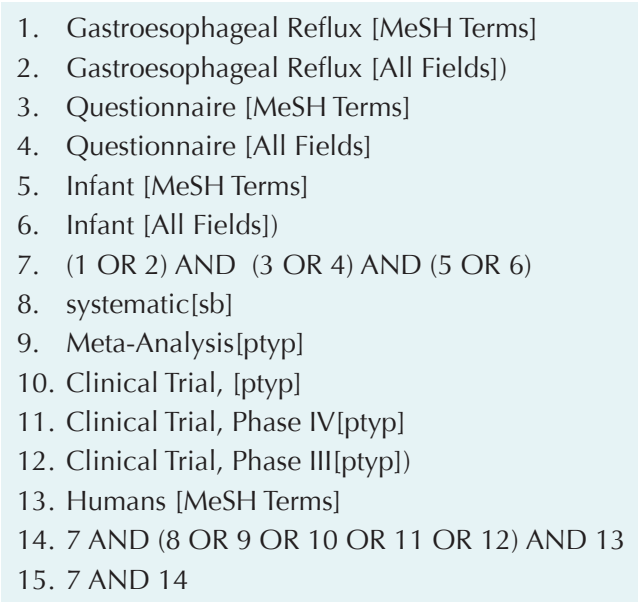

Fuente: elaboración propia.

nándose aquellos con información relevante al objetivo de la revisión; se seleccionaron estudios clínicos, revisiones sistemáticas y/o meta-análisis que evaluaran el uso de algún cuestionario como herramienta diagnóstica de los pacientes con signos y síntomas de ERGE. Solo se incluyeron estudios que estuvieran disponibles en texto completo, excluyendo cartas editoriales y resúmenes de publicaciones.

Extracción de los datos de estudios individuales

El contenido de todos los estudios clínicos seleccionados se recopiló en una base de datos inicial con la finalidad de decidir si los datos obtenidos eran combinables o no, para la posible realización de meta-análisis.

\section{Evaluación de la calidad de los estudios}

En los estudios seleccionados se buscó que cumplieran con la información requerida de la escala Newcastle-Ottawa, que está compuesta por ocho ítems, divididos en tres dimensiones (comparación, selección, tipo de estudio), la cual evalúa la calidad de los estudios de investigaciones de corte, transversales o caso-control. ${ }^{18}$

\section{RESULTADOS}

\section{Selección de los estudios}

Con la estrategia de búsqueda realizada (algoritmo de búsqueda), se encontró un total de 191 estudios de potencial interés (Figura 1). Después de eliminar los repetidos y leer los resúmenes, se decidió revisar un total de 14 textos completos, los cuales fueron analizados para su posterior evaluación. Finalmente, se seleccionaron tres estudios, el de Kleinman 2006, Deal 2005 y Stordal 2005.

\section{Características de los estudios}

El estudio de Kleinman es observacional multicéntrico, con un seguimiento de tres semanas, realizado entre noviembre 2002 y febrero 2003,

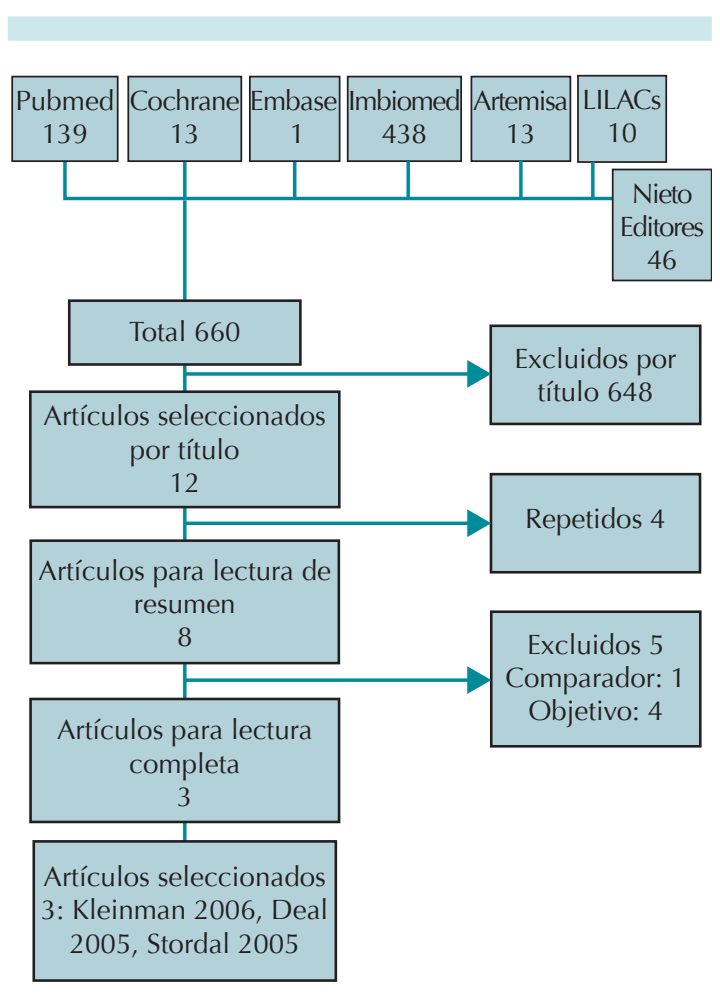

Figura 1. Algoritmo de PRISMA de la selección de la evidencia de la revisión sistemática.

Fuente: Elaboración propia 
en el que participaron 185 cuidadores de pacientes menores de 18 meses con diagnóstico de ERGE y 93 cuidadores de pacientes pediátricos que sirvieron como grupo control. El objetivo de dicho estudio fue revisar el cuestionario de reflujo gastroesofágico en lactantes (Infant Gastroesophageal Reflux Questionnaire [I-GERQ]) con base en la información de los padres de los niños con ERGE. Los cuidadores completaron el cuestionario cada semana y anotaron los síntomas en un diario cada día; tanto ellos como los médicos evaluaron la severidad de la enfermedad global y cambios en la sintomatología de ERGE. Finalmente, se obtuvo una confiabilidad interna para el I-GERQ que varió de 0.86-0.87 y una confiabilidad test-retest de 0.85 , demostrándose una diferencia estadísticamente significativa entre los casos y los controles $(p<0.01)$; las correlaciones con el diario fueron estadísticamente significativas $(p<0.05)$, la media de cambio de las puntuaciones en el IGER-Q mejoraron -5.7 versus -0.3 para los pacientes en los que los cuidadores reportaron con mejoría o sin cambio, respectivamente, y una media de cambio reportada por los médicos de -5.7 y - 0.1 para los pacientes con mejoría o sin cambio, respectivamente, ambas diferencias estadísticamente significativas $(p<0.001) .^{16}$

Por su parte, el estudio de Deal es piloto; en él, se desarrollaron y evaluaron dos cuestionarios en pacientes con y sin diagnóstico clínico de ERGE en una población de 1-11 meses y otra de 1-4 años. El cuestionario para el primer grupo fue el GERD Symptom Questionnaire-Infants (GSQ-I) y para el segundo grupo el GORD symptom questionnaire (GSQ-YC), ambas poblaciones tuvieron un grupo control, los padres o cuidadores de los niños completaron los cuestionarios respecto a la frecuencia y severidad de los síntomas, con una escala de 1 (no severo) a 7 (muy severo). La frecuencia de la sintomatología fue el número de ocurrencias en los 7 días previos al Ilenado del cuestionario. Los lactantes con ERGE tuvieron una prevalencia significativamente más alta que los controles $(\mathrm{p}<0.05)$, misma diferencia que se observó en los pre-escolares $(\mathrm{p}<0.05)$. Se calculó una puntuación individual para cada síntoma (ISS) y una promedio (CSS). Para los lactantes se encontró que una CSS $>27$, tuvo una sensibilidad de $90 \%$ para detectar a los pacientes con ERGE y especificidad de $83 \%$. En pre-escolares se encontró que una CSS $>8$, tuvo una sensibilidad de $85 \%$ para detectar a los pacientes con ERGE y una especificidad de $81.5 \% .{ }^{19}$

Por último, Stordal, en 2005, hizo su estudio con 99 niños entre 7 y 16 años con sospecha de ERGE, con un cuestionario de 7 ítems respecto a la sintomatología; una semana previa a un estudio de $\mathrm{pH}$ para validarlo y a su vez comparar el cuestionario y la pHmetría. El monitoreo de $\mathrm{pH}$ fue realizado en una sesión de 24 h, considerándose un índice de reflujo de 5 o más como anormal. Los pacientes con resultados del estudio de $\mathrm{pH}$ normales y anormales fueron comparados con la frecuencia de síntomas. Se encontró que 37 niños tuvieron estudio anormal de $\mathrm{pH}$ y 62 resultados normales; el mayor síntoma de discriminación fue regurgitación/vómito. Se encontró que 75\% de los niños con $\mathrm{pH}$ anormal y $44 \%$ tuvieron puntuación sintomática positiva (OR 3.78, IC 95\% $1.52-9.37 \%, p=0.006)$. El cuestionario generó una especificidad de $96 \%$ y una sensibilidad de $75 \%$, indicando que si la población general tuviera una prevalencia de ERGE de 5\% nos daría un valor predictivo positivo de $50 \%$ y un valor predictivo negativo de $98.6 \%$, por lo anterior, se concluye que existe una asociación débil entre los síntomas de reflujo y un estudio positivo de $\mathrm{pH}$ en niños de 7-16 años. ${ }^{14}$

\section{Calidad de los estudios}

Debido a que los estudios seleccionados fueron observacionales de casos y controles, el instrumento que se utilizó para evaluar la calidad fue la escala New-Castle Ottawa. Los tres estudios cumplieron con los criterios establecidos, respecto a la selección, la definición de los casos y 
los controles fue adecuada; en cuanto a la representatividad, se tomaron series de casos; sobre la comparabilidad los controles fueron seleccionados para evaluar la presencia de síntomas de ERGE y finalmente la exposición fue comprobada mediante la evaluación clínica de los pacientes.

\section{DISCUSIÓN}

El reflujo gastroesofágico ocurre en más de dos tercios en los lactantes menores de un año de edad, en el que un diagnóstico, tratamiento y referencia oportuna contribuirían a mejorar la situación clínica en los pacientes pediátricos. ${ }^{10}$ De tal forma que asegurar el diagnóstico permitirá un correcto tratamiento para los pacientes, así como evitar complicaciones y mejorar la calidad de vida; ${ }^{7}$ sin embargo, y pese a las diferentes herramientas diagnósticas, existen pocos instrumentos no invasivos para evaluar la progresión y remisión de la enfermedad en el tiempo y para evaluar los tratamientos en los ensayos clínicos. ${ }^{16}$ Uno de estos instrumentos es el cuestionario IGER-Q, el cual fue desarrollado y validado para evaluar los síntomas relacionados a la ERGE en niños, ${ }^{20,21}$ así como para obtener información clínica de los pacientes sobre la frecuencia y calidad de los síntomas, aunque se dice que la evaluación de la severidad es algo limitada. ${ }^{8}$

Es importante mencionar que existen pocas guías de práctica clínica para el tratamiento de ERGE en pediatría, tras la búsqueda exhaustiva en diversas bases de datos, solamente estuvieron disponibles la Guía de Diagnóstico y tratamiento del Reflujo Gastroesofágico en Pediatría de Cenetec $^{10}$ y la Guía de Enfermedad de Reflujo Gastroesofágico: reconocimiento, diagnóstico y manejo en niños y jóvenes, de NICE, ${ }^{22}$ las cuales dentro del abordaje diagnóstico no hacen mención alguna sobre el uso de cuestionarios para ERGE; también se encontró la Guía de la Asociación Española de Gastroenterología, la cual menciona el Patient Questionnaire y el Questionnaire for Gastroesophageal Reflux Disease, ${ }^{23}$ describe que son poco específicos o contienen demasiadas preguntas, ${ }^{24}$ es importante mencionar que ambos cuestionarios se utilizan en población adulta con ERGE y no se utilizan en pacientes pediátricos. También se encontró las guías NASPGHAN/ ESPGHAN publicadas en 2009,7 las cuales mencionan algunos cuestionarios que se han empleado en población pediátrica; sin embargo, se menciona que ninguno de estos ha sido validado en esta población. Llama la atención el hecho de que hasta el momento existen pocas guías clínicas publicadas para una enfermedad con una alta prevalencia en niños como lo es la ERGE y que no se describa el uso de cuestionarios como herramientas diagnósticas, puesto que podrían ser de utilidad en el primer nivel de atención donde no se cuenta de primera instancia con las herramientas técnicas diagnósticas y terapéuticas recomendadas en dichas guías.

Como pudo observarse en la revisión sistemática existen pocos estudios que evalúan el uso de cuestionarios para ERGE, dada la poca evidencia clínica disponible no fue posible realizar un meta-análisis para poder evaluar dicha información ya que los estudios encontrados emplean diferentes cuestionarios en poblaciones no homogéneas; por supuesto, la metodología y los resultados evaluados no pudieron analizarse de forma conjunta.

En México, se realizó un estudio por Villalpando y sus colaboradores en $2009^{25}$ para determinar la frecuencia de ERGE, utilizando el cuestionario evaluado por Stordal y su grupo, así como su asociación con asma y obesidad, reportando una frecuencia de hasta un $48 \%$ de síntomas gastrointestinales superiores cuando se presentan estas enfermedades de manera concomitante; sin embargo, no se hizo alguna prueba complementaria como 
pHmetría con o sin impedancia para el diagnóstico de ERGE.

La utilidad de los cuestionarios para determinar síntomas de ERGE es una práctica poco utilizada en niños; sin embargo, y por los múltiples factores que acompañan a la ERGE, existe controversia sobre las recomendaciones respecto a utilizar este instrumento de diagnóstico. Estos cuestionarios serían de utilidad para apoyo en el diagnóstico para los síntomas de ERGE en el primer nivel de atención en donde los estudios de gabinete no están disponibles, como se pudo observar la sensibilidad se mantuvo en un rango del $75 \%$ hasta el $90 \%$ y una especificidad desde $80 \%$ hasta un $96 \%$ por lo que podría apoyar en la selección de los pacientes con sospecha de ERGE que requieren un estudio de diagnóstico como pHmetría, la cual tiene una sensibilidad de $87-93.3 \%$ y especificidad de $92.9-97 \% .{ }^{26} \mathrm{Sin}$ embargo, el estudio que evalúa el cuestionario IGER-Q no reporta estos parámetros, solo indica que existió diferencia estadísticamente significativa entre el grupo de intervención y el control.

De acuerdo a los resultados obtenidos en las diferentes poblaciones evaluadas, se puede observar que a medida que aumenta la edad de los pacientes disminuyó la sensibilidad del cuestionario utilizado, así para pacientes de 1-11 meses se obtuvo 90\%, 1-4 años $85 \%$ y 7-16 años $75 \%$, en este caso se hubiera esperado lo contrario, ya que los pacientes de mayor edad pueden expresar por sí mismos la sintomatología que presentan y apoyar al diagnóstico, respecto a la especificidad para los primeros rangos de edad tuvo un valor de 83 y $81.5 \%$ comparado con $96 \%$ en pacientes de 7-16 años, en este caso con los resultados reportados se corrobora que a mayor edad en los pacientes es posible descartar un caso negativo cuando lo sea. Por otra parte, también se observó que no existe un cuestionario único para la medición de síntomas gastrointestinales superiores; si bien los tres estudios clínicos seleccionados ${ }^{14,16,19}$ para la realización de este análisis incluyeron diferentes edades entre la población pediátrica (lactantes, 1-4 años y 7-16 años), también investigan signos y síntomas específicos de ERGE $^{11,12,14,16,17}$ y en el $80 \%$ de los casos estos cuestionarios se basan en conocer la frecuencia y severidad de los síntomas principales en la población pediátrica como lo son pirosis, regurgitación y/o vómito dependiendo de la edad de presentación. ${ }^{7,8}$ De estos tres estudios clínicos, en el trabajo publicado por Stordal en 2005 fue en el único que se realizó una pHmetría posterior como estudio clínico complementario para el diagnóstico de la ERGE, mostrando una especificidad del 96\% y una sensibilidad de $75 \%$, lo cual aumenta la credibilidad del médico clínico en este cuestionario; sin embargo, en los resultados el autor concluye en una asociación débil entre los síntomas de reflujo y un estudio positivo de $\mathrm{pH}$ en niños de 7-16 años. ${ }^{14}$

Finalmente, es importante mencionar que el uso de cuestionarios para ERGE ha sido mayormente documentado en adultos; sin embargo, los resultados son variables y no permiten hacer una evaluación en conjunto, por lo que utilizarlos como herramienta diagnóstica de ERGE en niños implicaría un riesgo de sub-diagnosticar a los pacientes y por ende que éstos no reciban el tratamiento adecuado afectando su salud y calidad de vida.

\section{CONCLUSIÓN}

Por lo anterior, se concluye que el uso de los cuestionarios como herramienta diagnóstica de ERGE en pacientes pediátricos es escaso y la evidencia clínica disponible no es suficiente para realizar una recomendación apropiada, pretendemos que este documento sirva para fomentar la investigación clínica en el área permitiendo evaluar la utilidad de esta herramienta diagnóstica. 
Hernández-Pliego RR et. al. Revisión sistemática de cuestionarios para ERGE en niños

\section{Agradecimientos}

Este trabajo fue apoyado en parte por los programas de Consejo Nacional de Ciencia y Tecnología (CONACYT, México) y el programa Sistema de Administración de Programas y Proyectos de Investigación (SIP/IPN).

\section{REFERENCIAS}

1. Remes J, Rodríguez B, Valdovinos, M. La enfermedad por reflujo gastroesofágico en México. Revisión sistemática. Rev Gastroenterol Mex. 2006;712(2):151-9. http://www.imbiomed.com.mx/1/1/articulos.php?method=showDetail\&id_ articulo=36949\&id_seccion=50\&id_ejemplar=3820\&id_revista $=10$

2. Nandurkar S, Talley J. Epidemiology and natural history of reflux disease. Best Pract Res Clin Gastroenterol. 2000;14(5):743-57. http://www.ncbi.nlm.nih.gov/pubmed/10780569

3. Vakil N, Van Zanten V, Kahrilas P, Dent J, Jones R. Global Consensus Group. Montreal definition and classification of gastroesophageal reflux disease: a global evidence-based consensus. Am J Gastroenterol. 2006;101(8):1900-20. http://www.ncbi.nlm.nih.gov/pubmed/16928254

4. Huerta F, Tamayo J, Noble A, Remes J, Valdovinos M, Carmona R. Consenso mexicano de enfermedad por reflujo gastroesofágico parte I. Rev Gastroenterol Mex. 2012;77(4):193-213. http://www.revistagastroenterologiamexico.org/es/consenso-mexicano-enfermedad-porrelujo/articulo/S0375090612001322/

5. Nelson S, Chen E, Syniar G, Christoffel K.One-year follow-up of symptoms of gastroesophageal reflux during infancy. Pediatrics. 1998;102(6):E67. http://www.ncbi.nlm.nih. gov/pubmed/9832595

6. Campanozzi A, Boccia G, Pensabene L, Panetta F, Marseglia A. Prevalence and Natural History of Gastroesophageal Reflux: Pediatric Prospective Survey. Pediatrics. 2009;(123)3:779-83. http://pediatrics.aappublications. org/content/123/3/779.abstract

7. Vandenplas $Y$, Rudolph C, Di Lorenzo C, Hassall E, Liptak G, Mazur L, et al. Pediatric gastroesophageal reflux clinical practice guidelines: joint recommendations of the North American Society for Pediatric Gastroenterology Hepatology, and Nutrition (NASPGHAN) and the ESPGHAN. J Pediatr Gastroenterol Nutr. 2009;49(4):498-547. http://www. naspghan.org/files/documents/pdfs/position-papers/ FINAL\%20-\%20JPGN\%20GERD\%20guideline.pdf

8. Nelson P, Chen H, Syniar M, Christoffel K. Prevalence of symptoms of gastroesophageal reflux during infancy. A pediatric practice based-survey. Arch Pediatr Adolesc Med. 1997;151(6):569-72. http://www.ncbi.nlm.nih.gov/ pubmed/9193240
9. Sherman P, Hassall E, Fagundes U, Gold B, Kato S, Koletzko S, et al. A global, evidence based consensus on the definition of gastroesophageal reflux disease in the pediatric population. Am J Gastroenterol. 2009;104(5):1278-95. http:// www.ncbi.nlm.nih.gov/pubmed/19352345

10. Secretaría de Salud. Diagnóstico y tratamiento de reflujo gastroesofágico en pediatría en el primer nivel de atención. México, D.F. Secretaría de Salud, 2014. http://www. cenetec.salud.gob.mx/descargas/gpc/CatalogoMaestro/013_GPC_ERGEPED/SSA_013_08_EyR.pdf

11. Orenstein $\mathrm{S}$. Infantile reflux: different from adult reflux. Am J Med. 1997;103(5A):114S-119-S. http://www.ncbi. nlm.nih.gov/pubmed/9422635

12. Ashorn M, Ruuska T, Karikoski R, Laippala P. The natural course of gastroesophageal reflux disease in children. Scand J Gastroenterol, 2002;37(2):638-41. http://www. ncbi.nlm.nih.gov/pubmed/12126239

13. Hernández M, Ortega R, Márquez V. Reflujo gastroesofágico en pediatría ¿Cuándo amerita tratamiento?: Controversia de las últimas décadas.Rev Med Sur. 2008;15(4):288-94. http://www.medigraphic.com/pdfs/medsur/ms-2008/ ms084e.pdf

14. Stordal K, Johannesdottir G, Bentsen B, Sandvik L.Gastroesophageal reflux disease in children: Association between symptoms and $\mathrm{pH}$ monitoring. Scand J Gastroenterol. 2005:40(6):636-40. http://www.ncbi.nlm.nih.gov/ pubmed/16036522

15. Jones R, Junghard O, Dent J, Vakil N, Halling K, Wernersson $B$, et al. Development of the GerdQ, a tool for the diagnosis and management of gastro-oesophageal reflux disease in primary care. Aliment Pharmacol Ther. 2009;30(10):1030-8. http://www.ncbi.nlm.nih.gov/ pubmed/19737151

16. Kleinman L, Rothan M, Strauss R, Orenstein S, Nelson S, Vandenplas $Y$, et al. The Infant Gastroesophageal Reflux Questionnaire Revised: Development and Validation as an Evaluative InstrumentClin Gastroenterol Hepato. 2006;4(5):588-96. http://www.ncbi.nlm.nih.gov/pubmed/16678075

17. Orenstein S, Cohn J, ShalabyT, Kartan R. Reliability and validity of an infant gastroesophageal reflux questionnaire. Clin Pediatr (Phila).1993;32(8):472-84. http://www.ncbi. nlm.nih.gov/pubmed/8403746

18. Wells G, Shea B, O'Connell D, Peterson J, Welch V, Losos M, et al. Ottawa Hospital Research Institute. The NewcastleOttawa Scale (NOS) for assesing the quality of nonrandomised studies in metaanalyses. [En línea] 2014. [Citado el: 07 de 06 de 2015.] http://www.ohri.ca/programs/ clinical_epidemiology/oxford.asp.

19. Deal L, Gold B, Gremse D, Winter H, Peters S, Fraga D, et al. Age-specific questionnaires distinguish GERD symptom frequency and severity in infants and young children: development and initial validation. J Pediatr Gastroenterol Nutr. 2005;41(5):178-85. http://www.ncbi.nlm.nih.gov/ pubmed/16056096 
20. Orenstein R, Shaaby M, Cohn F. Reflux symptoms in 100 normal infants: diagnostic validity of the infant gastroesophageal reflux questionnaire. Clin Pediatr (Phila). 1996;35(12):607-14. http://www.ncbi.nlm.nih.gov/ pubmed/8970752

21. Rudolph D, Mazur J, Liptak S, Baker D, Boyle T, Colletti $B$, et al. Guidelines for evaluation and treatment of gastroesophageal reflux in infants and children: recommendations of the North American Society for Pediatric Gastroenterology and Nutrition. J Pediatr Gastroenterol Nutr. 2001;32(Supl 2): S1-31. http://www.ncbi.nlm.nih. gov/pubmed/11525610

22. National Institute for Health and Care Excellence.Gastrooesophageal reflflux disease: recognition, diagnosis and management in children and young people. United Kingdom: 2015. http://www.nice.org.uk/guidance/ng1/ resources/gastrooesophageal-reflux-disease-recognitiondiagnosis-and-management-in-children-and-youngpeople-51035086789
23. Manejo del Paciente con Enfermedad por Reflujo Gastroesofágico. Asociación Española de Gastroenterología, Sociedad Española de Medicina de Familia y Comunitaria y Centro Cochrane Iberoamericano.Barcelona: Elsevier Doyma, 2007. http://www.guiasgastro.net/guias_full/ textos/erge.pdf

24. Fraser A, Delaney B, Moayyedi P. Symptom-based outcome measures for dyspepsia and GERD trials: a systematic review. Am J Gastroenterol. 2005;100 (2):442-52. http:// www.ncbi.nlm.nih.gov/pubmed/15667506

25. Villalpando S, Ura J, De Río B, Heller S. Asociación de asma, obesidad y enfermedad por reflujo gastroesofágico en niños. Bol Med Hosp Infant Mex. 2009; 66 (Mar-Abr): 153-9. http://www.medigraphic.com/pdfs/bmhim/hi2009/hi092f.pdf

26. Velasco A. Actualización sobre Enfermedad por Reflujo Gastroesofágico en niños. Revista Colombiana de Gastroenterología. 2014;29(1):55-62. 